\title{
Toward a Taxonomy of Autonomic Sleep Patterns with Electrodermal Activity
}

\author{
Akane Sano and Rosalind W. Picard, Fellow, IEEE
}

\begin{abstract}
This paper presents a first version of a taxonomy of automatic sleep patterns found with the Affectiva $Q^{\mathrm{TM}}$ Sensor, a wireless, logging biosensor that measures skin conductance, skin temperature, and motion comfortably from the wrist. Several studies have examined electrodermal activity (EDA) during sleep, but they focused on an analysis of EDA for only a small number of nights. We quantitatively analyzed EDA during sleep in three study situations: (1) Comparing EDA with polysomnography (PSG) from seven subjects in a sleep lab, (2) Characterizing multiple nights of EDA in a sleep lab, in a hospital and at home from 24 subjects, and (3) Gathering long-term EDA (30-60 nights) patterns from three subjects during home sleep. After gathering this rich corpus of data, we characterized inter- and intra- individual differences of EDA features and the relation of EDA peaks to subjective sleep quality. Here we present results from the three studies in an effort to begin to characterize autonomic patterns found in natural sleep.
\end{abstract}

\section{INTRODUCTION}

$\mathrm{E}^{\mathrm{le}}$ lectrodermal activity (EDA) is widely used in psychophysiology and provides a measure of sympathetic nervous system (SNS) activity, where the SNS is one of the main branches of the autonomic nervous system. Classically, EDA has been measured as skin conductance and involves attaching wired and gelled electrodes to the skin [1]. Studies on EDA during sleep have shown that EDA is more likely to appear elevated with high frequency "storm" patterns during deep sleep [2], that EDA can distinguish wake and sleep and indicate sleep onset, and that it is not generally sufficient for identifying sleep stages [3].

These studies focused on analysis of only one night or on short-term data with only limited features, and to our knowledge all of the studies were performed in sleep labs where sleep may not have been as natural as at home. In order to characterize autonomic sleep patterns precisely and quantitatively, long-term natural data is needed. This paper is the first we know of to present long-term EDA data and an initial characterization of the patterns in this SNS signal.

Polysomnography (PSG) is currently a gold standard to evaluate and diagnose sleep patterns; however, the sensors tend to be uncomfortable and expensive, take a long time to put on, and may interfere with home sleep. Actigraphy is a

Manuscript received April 13, 2011. This work was supported in part by the MIT Media Laboratory Consortium sponsors.

Akane Sano is a graduate student and Rosalind W. Picard is a professor in the Affective Computing Group, Media Lab, Massachusetts Institute of Technology, Cambridge, MA, 02139, USA (e-mail: akanes@mit.edu). Full disclosure: Picard is also co-founder, chief scientist, and chairman of Affectiva, the company who manufactures the $\mathrm{Q}^{\mathrm{TM}}$ sensors we used. much less invasive method often used to evaluate daytime and sleep activity with a wrist device; however, it is limited to measurement of movement, and can suggest you are asleep even when you are awake, e.g., if you are engrossed watching a great movie and not moving. The sensor used in this study measures both EDA and actigraphy, so it can more accurately measure sleep in detail.

This paper aims to evaluate EDA sleep patterns quantitatively in detail from healthy groups using the wearable sensor, and thereby begin to develop a taxonomy of autonomic patterns during sleep. We also analyze the relationship between this new EDA measurement from the wrist and standard PSG. One of our goals is to better understand what the changing patterns of EDA mean in terms of traditional PSG.

\section{METHODS}

\section{A. Measurement}

In this paper, we examine EDA during sleep by monitoring skin conductance (SC) on the inner wrist (ventral forearm) using the Affectiva $\mathrm{Q}^{\mathrm{TM}}$ Sensor with $1 \mathrm{~cm}$ diameter $\mathrm{Ag}-\mathrm{AgCl}$ dry electrodes. EDA, actigraphy and skin surface temperature data are logged at the sampling rate, either at $8 \mathrm{~Hz}$ or $32 \mathrm{~Hz}$. The Massachusetts Institute of Technology Committee On the Use of Humans as Experimental Subjects (COUHES) approved this study. We collected EDA under 3 different conditions: (1) EDA data with concurrent polysomnography (PSG) consisting of 30-second epochs of sleep stages labeled by experts (Wake, REM, Non-REM 1, 2 and Slow wave sleep (SWS)) for seven healthy university students (ages 18-22, 5males) each sleeping one night in a hospital; (2) 54 nights of EDA data for 24 healthy university students (ages 18-22, 16 males) sleeping at hospital, laboratory and home and (3) long-term EDA data $(26,66$, and 48 nights) for three subjects (one male and two females, ages 27, 30 and 48) sleeping at home. Subjects put the sensor on the wrist before going to bed, and took it off after waking.

\section{B. Analysis}

The data are analyzed as follows:

1. Pre-processing: Standard zero-crossing and Cole's function applied to the accelerometer data was used to discriminate between sleep and wake [4]. Only data that is determined to be sleep was further processed. EDA data was low-pass filtered (cutoff frequency $0.4 \mathrm{~Hz}, 32 \mathrm{nd}$ order FIR filter).

2. We detected EDA "storm" regions, where "storm" (first described by Burch [5]) refers to a region of EDA with a burst 
of high frequency peaks. We obtained the first derivative of filtered EDA and determined where the slope exceeds a value of 0.09 micro Siemens per second.

Burch originally quantified a storm as a minimum of five galvanic skin responses (GSRs)/min for at least ten consecutive minutes of sleep. In this paper, we defined "storm epochs" as a 30 second epoch with a minimum of three GSRs/30s. If storm epochs are adjacent or within five minutes of each other, they are combined into a "storm".

3. We counted the number of storms per night as well as the number of storm epochs. We also calculated the number of peaks in storms, durations of storms, peak frequency, amplitude and onset time of the first storm. We compared these storm characteristics to sleep stages.

4. We analyzed the correlations between EDA characteristics and sleep stages. We also analyzed correlations among EDA characteristics.

5. Participants in the first two studies were also asked to rate their sleep quality (scale $=1-4$ : Excellent, Good, Fair, and Poor) and we compared this to the EDA storm patterns.

\section{RESULTS}

\section{A. Relationship between PSG and EDA}

Figure 1 shows one night of EDA, motion and EDA storms with sleep stages and Fig. 2 shows the percentage of each sleep stage over one night. As shown in Fig. 3, six out of seven subjects showed EDA storms during the night. Most storms occurred in Non-REM 2 and SWS. In most subjects, SWS showed more variation of EDA amplitude and most subjects showed less than one micro Siemens EDA (Fig. 4).

We examined the correlation between EDA characteristics and sleep stages. A higher percentage of storm epochs during SWS of the 1st quarter of the night was associated with slower frequency of EDA peaks during SWS over the whole night $(\mathrm{p}<.01)$ and with greater subjective sleep quality $(\mathrm{p}<$ .05 , Fig. 5). Higher average EDA peaks per minute over the night was correlated with higher average EDA peaks per minute in SWS $(\mathrm{p}<.01)$.

\section{$B$. Inter-individual differences in EDA}

We analyzed the correlations between features, such as frequency of peaks and storms, and the time until onset of the first storm.

According to all data from 24 subjects, 36 out of 54 nights did show storms (67\%). Analysis showed earlier onset of the first storm was correlated with a larger number of storms $(\mathrm{p}<$ .05 ). A longer percentage of storms in the 1st quarter of the night was associated with a larger number of peaks over the night $(\mathrm{p}<.01)$. In addition, we found that nights with two storms were most frequent (Fig. 6) and the average number of peaks in EDA storm epochs was most typically four (Fig. 7). Thirty seconds was most frequent in EDA storm duration; moreover, the longest duration was 51 minutes. Twenty peaks of storms were most often observed over the night (Fig. 8). The numbers of epochs with peaks and storms were not correlated with the length of sleep. The first storm occurred most often within 60 minutes after falling asleep (Fig. 9).

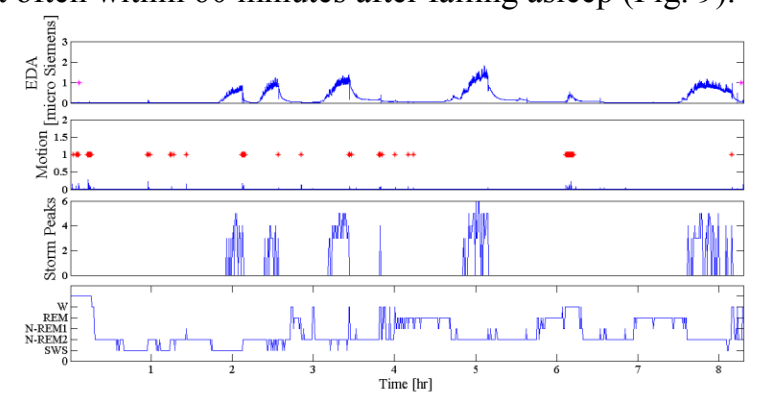

Fig. 1. EDA with motion, EDA storms and sleep stages

Pink marks on the first row indicate sleep and wake timing and red marks on the second row indicate wake moments from actigraphy
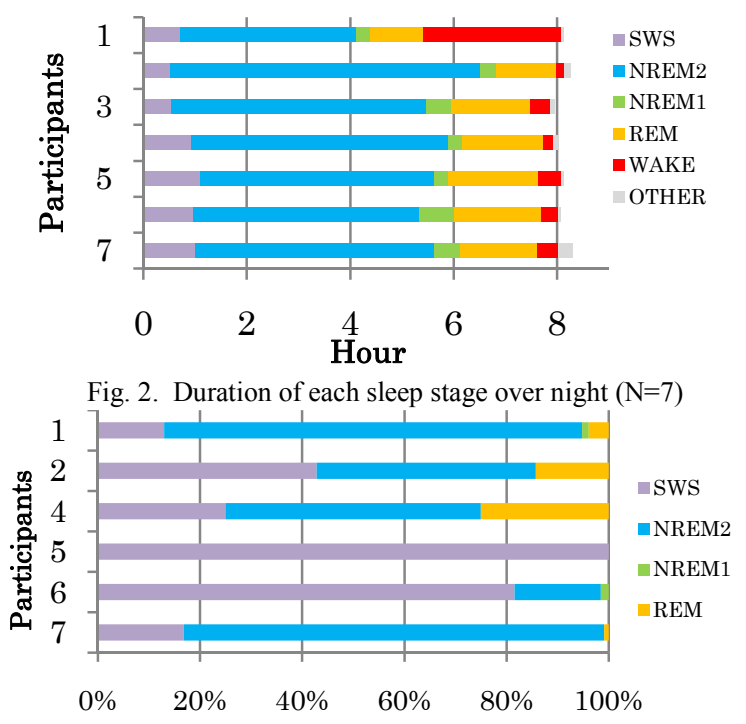

Fig. 3. Percentage of epochs having more than three peaks in 30 seconds, in each stage of sleep (in 6 out of 7 participants). The number on each graph is the percent of that night that included such storm epochs.

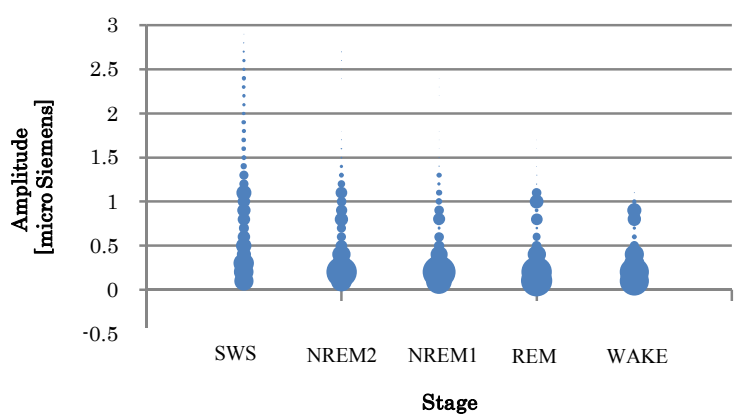

Fig. 4. Histogram of EDA amplitude in each sleep stage $(\mathrm{N}=7)$

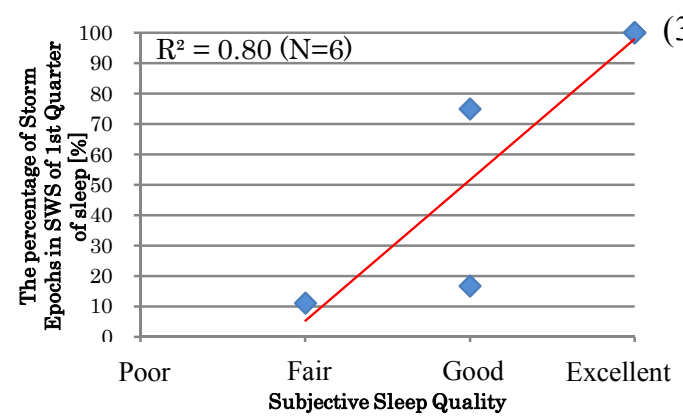

Fig.5. Correlation between subjective sleep quality and the percentage of storm epochs in SWS of the $1^{\text {st }}$ Quarter of sleep $(\mathrm{N}=6)$ 


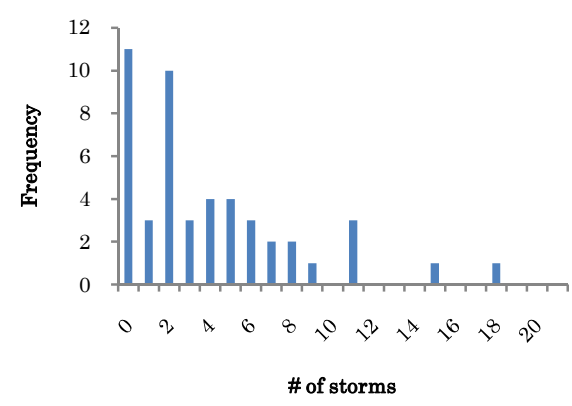

Fig. 6. Histogram of number of storms over night (54 nights)

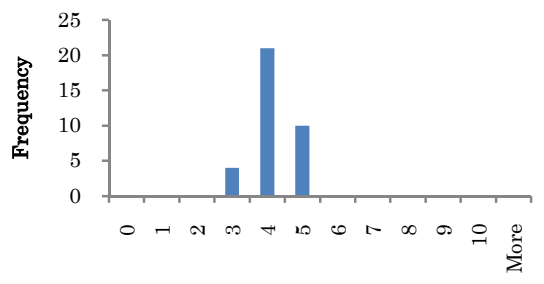

Average\# of peaks in storm epochs

Fig. 7. Histogram of average frequency of peaks in storm epoch (36 nights)

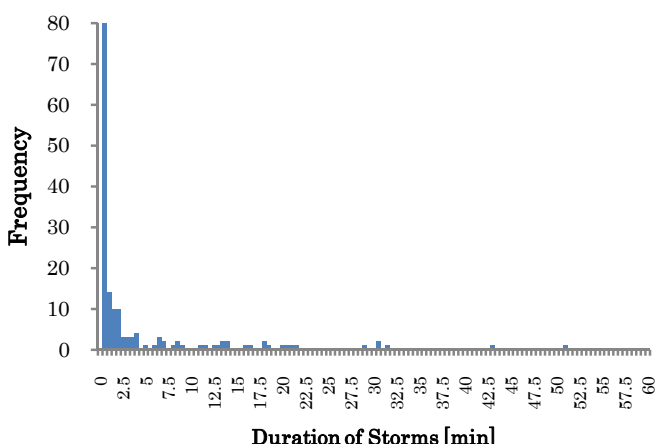

Fig. 8. Histogram of duration of EDA storms (36 nights, 161 storms)

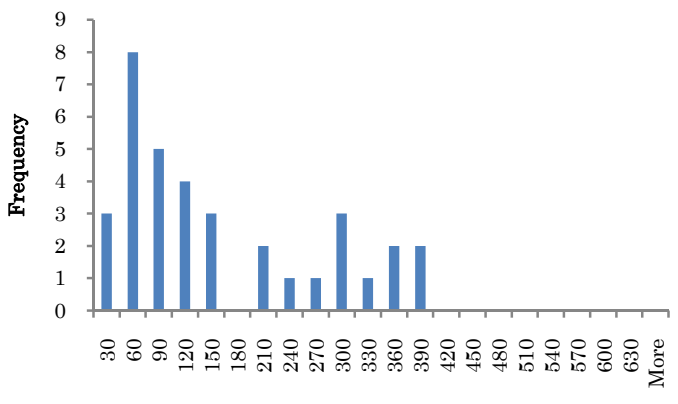

Onset of First Storm [min]

Fig. 9. Histogram of onset of first storm (36 nights)

\section{Intra/inter individual differences from long-term EDA}

We also analyzed long-term data from three subjects. As shown in Figure 10, participant 3 showed later sleep time and larger variation than participants 1 and 2 . However, the time initiating sleep was neither correlated with the onset of first storms nor the number of peaks of the first storm.

The three subjects showed storms in $64 \%, 77 \%$ and $71 \%$ of all nights. Figure 11 shows that the onset of the first storm was most probable within 60-120 minutes of sleep onset.
EDA amplitude was most likely to be less than 1.5 micro Siemens, as shown in Fig. 12. Figure 13 showed the percentage of epochs with peaks in quarters. Participant 1 showed the most storms in the first and the second quarter; on the other hand, participant 2 showed the majority of storms in the 3nd and 4th quarters and participant 3 showed storms equally in the 2nd, 3nd and 4th quarters. Moreover, participant 1 , who was on the most regular sleep schedule, showed that the number of peaks of EDA storms decreased as the number of storms increased (Fig.14). Long-term EDA data from three subjects also showed that earlier onset of the first storm is associated with a larger number of storms $(\mathrm{p}<$ .05 ), the same behavior seen with the six subjects in section A who showed storms.

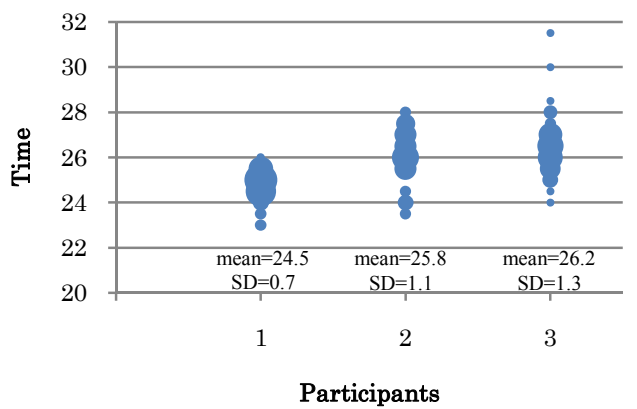

Fig. 10. Histogram of Sleep Start Time ( $\mathrm{N}=3$, home sleep)

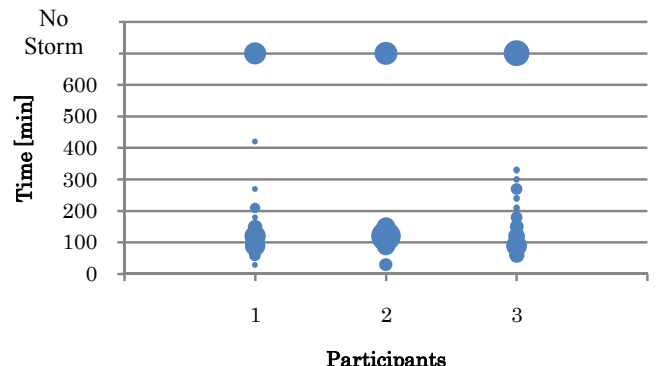

Fig. 11. Histogram of Onset of the first storm ( $N=3$, home sleep)

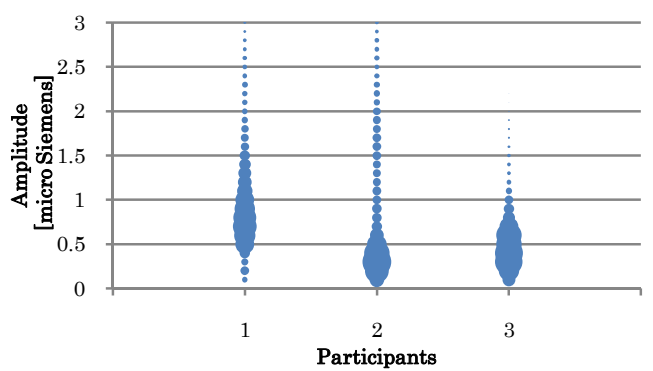

Fig. 12. Histogram of EDA amplitude ( $N=3$, home sleep)

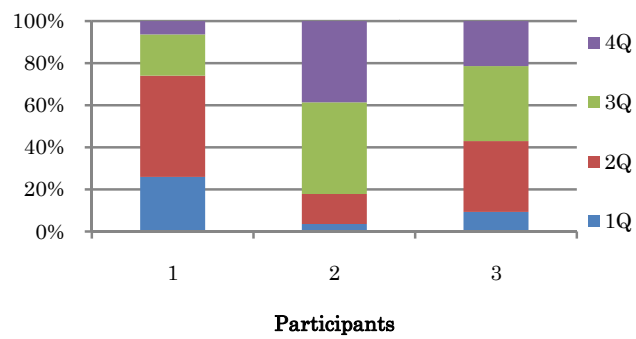

Fig. 13. Percentage of storm epochs in each quarter of sleep $(\mathrm{N}=3$, home sleep) 


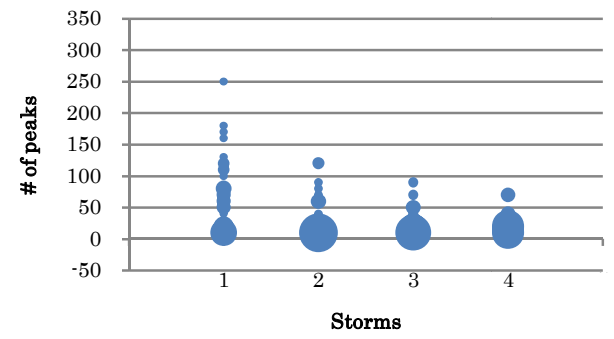

Fig. 14. Histogram of the number of peaks of EDA storms for participant 1

\section{DISCUSSION}

In this EDA study, we found intra- and inter-individual differences, but we also discovered some agreement and disagreement with previous findings.

EDA storms did not always occur every night. Moreover, EDA storms did not occur during every SWS and N-REM 2 stage; however, the occurrence tendency of EDA storms is consistent with previous reports that most spontaneous EDA storms occur during SWS and tend to decrease dramatically during REM sleep [6]. Moreover, this result can also explain another of our findings: higher average EDA peaks per minute over the night was correlated with higher average EDA peaks per minute in SWS $(p<.01)$. The most typical number of storms of 2 storms per night is consistent with the average of 2-3 storms per night shown previously [7]; however, Ware et al. reported that all subjects stormed on at least one night of four nights.

In our result, EDA storms also sometimes appeared in N-REM 2 and very rarely but sometimes briefly in REM.

For some subjects, the number of peaks in EDA storms decreased over the sleep period on most nights; however, this is not applicable to all subjects. Some studies have reported that EDA during sleep is related to presleep activity, stress, and sleep deprivation; however, clear and consistent findings have not been provided [8].

In addition, it is interesting to note that a higher percentage of storm epochs during SWS of 1st quarter of the night was associated with greater subjective sleep quality $(\mathrm{p}<.05)$. More synchronized subject data is needed to be confident of the findings, but this finding has the potential to enable the use of EDA peaks for biomarkers of subjective sleep quality.

Our results showed that one of the three participants, the one with the most regular bedtime, showed frequent peaks earlier during sleep, but the other two participants showed them later. Generally, more SWS occurs in the earlier part of the night than the later; therefore, this finding might be related to the fact that storms were observed in N-REM2 as well as SWS. Previous studies have shown that EDA is not evenly distributed over the night: Freixa i Baqué et al. found that spontaneous EDA activity is less frequent (i.e., 60\%) during the first full sleep cycle compared to the later three cycles [9]. However, at least one study has found that EDA was less frequent in the latter half of sleep, especially after the third full sleep cycle [10].

Finally, our EDA was measured on the ventral forearm, while previous studies mostly examined EDA on the palmar surface. Some of our earlier comparisons showed that the
EDA amplitude and storm patterns are usually more pronounced on the wrist than on the palm, and thus peaks are more likely to be found on the wrist [11]. These measures included over 100 nights and were on a small number of people so it is possible they do not hold across the population.

\section{CONCLUSION}

We analyzed EDA with PSG from hospital, laboratory and home sleep over many people and over many nights, focusing on the detection of high-frequency EDA peaks and regions of peaks called "storms." The occurrence and characteristics of EDA storms differed in each night with each subject; however, we found some tendencies: EDA storms tend to appear in N-REM 2 and SWS; around $70 \%$ of nights show EDA storms with an average of two storms and 30-60 minutes onset time to the first storm. Earlier onset of the first storm is related to a larger number of storms over the night. We also found a relationship between subjective sleep quality and a higher percentage of SWS storm epochs during the 1st quarter of the night.

\section{ACKNOWLEDGMENT}

The authors would like to thank Robert Stickgold and Hilary Wang, as well as all participants who cooperated in collecting EDA and PSG data.

\section{REFERENCES}

[1] W. Boucsein, "Electrodermal Activity (The Springer Series in Behavioral Psychophysiology and Medicine)," Springer,1992

[2] K. Asahina, "Paradoxical phase and reverse paradoxical phase in human sleep," Journal of Physiological Society of Japan, vol. 24, pp.443-450, 1962

[3] A. J. R. Koumans, B. Tursky, and P. Solomn, "Electrodermal levels and fluctuations during normal sleep," Psychophys»iology, vol. 5, pp. 300-306, 1968

[4] R. J. Cole, D. F. Kripke, D. J. Gruen W. Mullaney, and J. C. Gillin, "Automatic sleep wake identification from wrist activity," Sleep, vol.15, pp.461-469, 1992

[5] N. Burch, "Data processing of psychophysiological recordings," In L. D. Proctor \& W. R. Adey (Eds.), Symposium on the analysis of central nervous system data using computer methods, pp. 165-180,Washington, D.C.: National Aeronautics and Space Administration, 1965

[6] R. J Broughton, R. Poiré, \& C. A. Tassinari, "The electrodermogram (Tarchanoff effect) during sleep," Electroencephalography and Clinical Neurophysiology, vol.18, pp.691-708, 1965

[7] J. C. Ware, I. Karacan, R. B. Mefford, M. Hirshkowitz, R. L. Roessler, N. Kaya, and R. L. Williams, "Electrodermal activity during sleep: Relation to other polysomnographic parameters," Sleep Research, vol. 8, pp.73, 1979

[8] B. K. Lester, N. R. Burch, and R.C. Dossett, "Nocturnal EEG-GSR profiles: The influence of presleep states," Psychophysiology, vol.3, pp.238-248, 1967

[9] E. Freixa i Baqué and M. deBons, "Electrodermal asymmetry during human sleep, " Biological Psychology, vol. 17, pp. 145-151, 1983

[10] T. Hori, A. Miyasita, and Y. Niimi, "Skin potential activities and their regional differences during normal sleep in humans," Japanese Journal of Physiology, 20, pp. 657-671, 1970

[11] R.W. Picard, A. Sano and R. el Kaliouby, "Palmar vs. Forearm EDA during Natural Sleep at Home," 50th annual meeting of Society for Psychophysiological Research, Portland, OR, USA, Sep.29 - Oct.3, 2010 